\title{
THE EFFECTS OF CHANGE IN THE SAFETY ROPE PROTOCOL, HOLD SIZE, AND REST PERIOD ON PSYCHOPHYSIOLOGICAL RESPONSES IN SPORT ROCK CLIMBERS
}

original paper

( ) University School of Physical Education in Wroclaw

DOI: https://doi.org/10.5114/hm.2019.79733

\section{ARTUR MAGIERA, IWONA ŁUKASIK, ROBERT ROCZNIOK, OSKAR PLACEK}

The Jerzy Kukuczka Academy of Physical Education, Katowice, Poland

\section{ABSTRACT}

Purpose. The aim of the study was to evaluate the effects of a decrease in hold size, change in the safety rope protocol, and rest period shortening on heart rate (HR), heart rate variability (HRV), pre-climb anxiety, and perceived workload in sport rock climbers under laboratory settings.

Methods. A total of 12 intermediate sport rock climbers were recruited. The participants completed 2 routes of different climbing difficulty. They repeated the difficult route with a short 5 -minute rest period 3 times and repeated the same route with a change to lead climb. HR, HRV, anxiety level (Competitive State Anxiety Inventory-2 Revised), and climbing workload

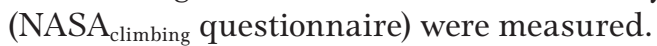

Results and conclusions. It was lead climb that elicited the most noticeable psychophysical response in the intermediate climbers. Prior to the climb, the performers exhibited marked sympathetic activation and higher cognitive anxiety. Climbing $\mathrm{HR}_{\text {peak }}$ was correlated with physical workload, psychological demand, and increasing fatigue; hence, it can be considered a variable that reflects the accumulative psychophysiological stress. $\mathrm{NASA}_{\text {climbing }}$ score effectively reflected differences in physical load (physical demand - strength and endurance) and psychological load (higher mental demand, temporal demand, and satisfaction level) during climbing.

Key words: rock climbing, heart rate, heart rate variability, anxiety, perceived climbing workload

\section{Introduction}

Rock climbing is a unique cognitive and physical challenge, practised on both natural rocks and artificial indoor climbing walls [1]. Nowadays, it has become a recreational activity, a form of active sport tourism, and a professional sport discipline. Its most popular subdiscipline, commonly referred to as sport rock climbing, is practised on climbing routes with permanently installed anchors. Owing to improvement in belay techniques, sport rock climbers can explore more difficult terrain and focus on the physical and technical aspects of climbing moves [2]. Sport rock climbing requires an individual to move vertically or horizontally via a series of hand- and/or footholds to form a route or climbing task. The physical and technical complexity of a climbing route is represented in the rating scale of difficulty (RSD) $[1,3]$ The difficulty of a climbing route is subjectively assessed. Each climber's assessment is based on their skill and climbing experience. It might be speculated that climbers give RSD judgements by comparing a particular route with those they did before, already rated by the first ascensionist [3]. Route difficulty, climbing style (on-sight [OS] ${ }^{1}$, red point [RP], Flash), and belay technique (lead or top rope climbing) pose different physical and technical demands and can therefore evoke a wide variety of mental and physiological responses [4-6].

Several researchers have attempted to determine the psychophysiological stress associated with rock climbing [4, 7-10]. Pre-climb anxiety and perception

\footnotetext{
${ }^{1}$ On-sight style (OS) - climbing on the first attempt, with no beta (prior knowledge) of the route.
}

Correspondence address: Artur Magiera, The Jerzy Kukuczka Academy of Physical Education, Department of Active Sport and Adventure Tourism, ul. Mikołowska 72a, 40-065 Katowice, Poland, e-mail: a.magiera@awf.katowice.pl

Received: April 19, 2018

Accepted for publication: November 14, 2018

Citation: Magiera A, Łukasik I, Roczniok R, Placek O. The effects of change in the safety rope protocol, hold size, and rest period on psychophysiological responses in sport rock climbers. Hum Mov. 2019;20(2):34-43; doi: https://doi.org/10.5114/ hm.2019.79733. 
of the workload after the climb were measured with the use of psychological inventories, including Competitive State Anxiety Inventory-2 Revised (CSAI-2R) and NASA Task Load Index (NASA-TLX). Physiological response to this type of stress mainly consists of autonomic nervous system stimulation, which can be estimated by heart rate (HR) and heart rate variability (HRV). HRV helps measure the balance/imbalance of the sympathetic nervous system and parasympathetic nervous system [11]. HR recording may allow objective monitoring of the psychophysiological stress.

The main aim of the study was to analyse HR, HRV, as well as anxiety (CSAI-2R) and perceived climbing load (NASA $\mathrm{N}_{\text {climbing }}$ ) in order to determine physical workload (different hold size) and the effects of short-term fatigue (different rest period) and psychological demand (different safety rope protocol) prior to and during indoor climbing.

\section{Material and methods}

\section{Participants}

The study group comprised 12 intermediate sport climbers ( 5 women and 7 men; level of climbing performance: $5 \mathrm{a}-6 \mathrm{~b}$ OS). The mean age of the participants was $23 \pm 4.6$ years, body weight $63 \pm 8.6 \mathrm{~kg}$, body height $171 \pm 6.7 \mathrm{~cm}$; they had $1-6$ years of climbing experience. All subjects received detailed information on the study procedures, aims, and risks.

\section{Experimental design}

The study consisted of 3 phases separated by recovery periods of at least 48 hours (Figure 1):
- Phase I: getting acquainted with the study procedure; responding to the Sport Competition Anxiety Test (SCAT) questions.

- Phase II: part 1 - each participant completed top rope 2 routes of different climbing difficulty (easy/difficult routes [II-ER $\left.\mathrm{TR}_{\mathrm{TR}} / \mathrm{II}-\mathrm{DR}_{\mathrm{TR}}\right]$ - effect of physical workload) with a $>20$-minute recovery period in between; part 2 - the climbers were asked to complete the difficult route 3 times with a short 5 -minute rest period

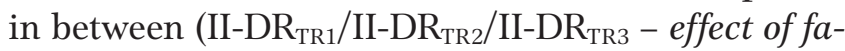
tigue).

- Phase III: repetition of part 1 of phase II in order to determine its reliability (III-ER $\mathrm{ER}_{\mathrm{TR}} / \mathrm{III}-\mathrm{DR}_{\mathrm{TR}}-$ test-retest) and, subsequently, lead repetition of the difficult route (III-ER $\mathrm{TR}_{\mathrm{TR}} / \mathrm{III}-\mathrm{DR}_{\mathrm{L}}$ - effect of psychological demand).

Each phase was preceded by a 10-minute warm-up. In order to minimize other environment influences, the experimental conditions were precisely determined - the same time of day, similar temperature and humidity in the indoor climbing settings.

\section{Measurements}

\section{Measurement and analysis of $H R$ and $H R V$}

HR and HRV were recorded with the Polar V800 heart rate monitor, characterized by high quality and measurement reliability [12].

HR was monitored throughout the study. Prior to the climb, 3-minute HR taken in a sitting position was analysed and the average HR was calculated (P$\mathrm{HR}_{\mathrm{ave}}$ ). HR was taken again immediately before the climb $\left(C L-\mathrm{HR}_{\text {start }}\right)$. During the climb, the average climb HR (CL-HR ave) and peak climb HR (CL-HR peak $)$ were determined.

\begin{tabular}{|c|c|c|c|c|c|c|c|}
\hline Phase I & getting acquaintec & udy procedure & & & & & \\
\hline \multirow{5}{*}{ Phase II } & & \multicolumn{4}{|c|}{ Easy route (II-ERTR) } & & 8 \\
\hline & & \multicolumn{4}{|c|}{ Difficult route (II-DRrR) } & & 5 \\
\hline & & \multirow{2}{*}{\multicolumn{3}{|c|}{ Difficult route (II-DRTrI) }} & 2 & & \\
\hline & & & & & S & & \\
\hline & & \multicolumn{3}{|r|}{ Difficult route (II-DRTss) } & & & \\
\hline \multirow{3}{*}{ Phase III } & & \multicolumn{3}{|r|}{ Easy route (III-ERTR) } & & & 8 \\
\hline & & & & Difficult route (III-DRrR) & & $\cdots$ & 5 \\
\hline & & \multicolumn{4}{|c|}{ Leading diff. route (III-DRL) } & & \\
\hline Activity & Rest (sitting) & $\begin{array}{c}\text { Arousal } \\
\text { determination } \\
\text { (sitting) }\end{array}$ & $\begin{array}{c}\text { Preparing for } \\
\text { the climb } \\
\text { (standing) }\end{array}$ & Climbing workload & $\begin{array}{c}\text { Short recovery } \\
\text { (sitting) }\end{array}$ & $\begin{array}{l}\text { Long recovery } \\
\text { (sitting) }\end{array}$ & \\
\hline Measurement & CSAI- $2 R$ & Pre-HRave, HRV & Cl-HRstart & Cl-HRave, Cl-HRpeak & NASAclinbing & & \\
\hline Time & $5 \min$ & $3 \min$ & $1-2 \min$ & $4 \min$ & $1-3 \min$ & $\geq 20 \min$ & \\
\hline
\end{tabular}

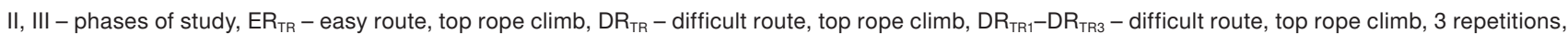
$\mathrm{DR}_{\mathrm{L}}$ - difficult route, lead climb

Figure 1. Study flow diagram 
A. Magiera, I. Łukasik, R. Roczniok, O. Placek, Psychophysiological responses in climbers

Short-term HRV was measured and analysed in accordance with the recommendations of the Task Force of the European Society of Cardiology and the North American Society of Pacing and Electrophysiology [13]. The measurements were performed under stable conditions, i.e., with the climbers in a sitting position, not talking, and not engaged in any energetic action. The data were recorded in the Polar V800 heart rate monitor to the nearest $1 \mathrm{~ms}$; artefacts were detected and corrected with the Kubios-HRV 2.2 software (Department of Physics, University of Kuopio, 2008). A 3-minute segment with the least amount of artefacts was selected from a 5-minute recording; the amount of artefacts was $<1 \%$ in each study subject. A detrending algorithm was implemented with the smoothness priors approach; very low frequency was deleted to focus on the power of low (LF) and high (HF) frequency components. Autoregressive model was used to estimate the power spectral density of R-R intervals.

Only the frequency domain parameters of HRV were analysed [13]:

- $\operatorname{lnTP}\left(\mathrm{ms}^{2}\right)$ : natural logarithm of total power of power spectral density for the analysed normal-to-normal intervals between consecutive heartbeats;

- $\operatorname{lnHF}\left(\mathrm{ms}^{2}\right)$ : natural logarithm of HF power (0.15$0.4 \mathrm{~Hz}$ ), reflecting modulation of parasympathetic tone by breathing and blood pressure changes;

- $\operatorname{lnLF}\left(\mathrm{ms}^{2}\right)$ : natural logarithm of LF power $(0.04-$ $0.15 \mathrm{~Hz}$ ), reflecting modulation of both sympathetic and parasympathetic tone;

- LF/HF ratio $(n)$ - the ratio between the power of LF and HF bands; a LF/HF ratio > 1 indicates the dominance of sympathetic over parasympathetic influences on HR.

\section{Pre-climb anxiety measurements}

Pre-climb cognitive anxiety was evaluated with the Polish version of CSAI-2R [14]. The climbers responded to questions on the occurrence or non-occurrence of a given pre-climb psychophysical experience using a 4-point response scale: 1 - not at all, 2 - somewhat, 3 - moderately so, and 4 - very much so. The inventory is based on the multidimensional anxiety theory by Martens, distinguishing between cognitive anxiety (Acog), somatic anxiety (Asom), and self-confidence (Scon) [14].

The Polish version of SCAT, a 15-item inventory that measures an athlete's anxiety levels while competing in their sport [14], was used to define trait anxiety.

\section{Post-climb analysis of perceived workload}

An analysis of perceived climbing workload was based on the NASA-TLX scores [15]. The test consists of 2 parts. Part 1 requirement is to evaluate the contribution of each of the 6 specified subscales to the workload of a specific task; the contribution is based on 15 pairwise comparisons of the 6 subscales. The second requirement is to provide numerical ratings on a 20-point scale reflecting the magnitude of each factor in this task [15]. NASA-TLX was used in rock climbing before [10] but was modified in our study to better describe the characteristics of the physical activity under investigation; from now on, the modification is referred to as $\mathrm{NASA}_{\text {climbing. }}$. The following factors were assessed: mental demand (Mental; How mentally demanding was the climb?), physical demand (strength) (Strength; How physically demanding was the crux?), temporal demand (Temporal; How hurried was the climb?), satisfaction level (positive emotions) (Satisfaction; How satisfied were you with completing the route?), physical demand (endurance) (Endurance; How hard did you have to work?), and frustration level (negative emotions) (Frustration; What was the level of negative emotions during the climb?).

\section{Changes in physical workload}

In phase II, change in the climbing workload was obtained through 2 different hold sizes (easy/difficult routes, II-ER $\mathrm{TR}_{\mathrm{TR}} / \mathrm{II}-\mathrm{DR}_{\mathrm{TR}}$ ). Two rows of handholds, including jugs (held with the entire hand, 4 fingers, 3 phalanges) and 2-finger pockets (2 fingers and distal phalanges), were fixed on a 7-meter high artificial climbing wall with the wall angle set at $95^{\circ}$. On the easy route, the climbers used the jugs as handholds and the jugs and 2-finger pockets as footholds. The difficult route only differed in that the 2-finger pockets served as handholds. The climbers repeated identical 8-second moves, climbing with a constant velocity the entire wall length twice (the descent lasted 16 seconds, the completion of the whole route $240 \mathrm{sec}-$ onds); from now on, this sequence will be referred to as a climbing task.

\section{The effect of short-term fatigue}

The recovery period between climbing tasks (> 20-minute) was usually sufficient for climbers. However, in the second part of phase II, the participants were asked to complete the difficult route 3 times with a 5-minute rest period in between, which was not a suf- 
ficient amount of recovery time (II-DR $\mathrm{TR}_{1} / \mathrm{II}_{-}-\mathrm{DR}_{\mathrm{TR} 2} /$ II-DR $\left.{ }_{\mathrm{TR} 3}\right)$. This part was designed to show the effect of short-term fatigue.

\section{Test-retest}

In phase III, the repetition of part 1 of phase II served to determine its reliability (III-ER $\mathrm{ER}_{\mathrm{TR}} / \mathrm{III}-\mathrm{DR}_{\mathrm{TR}}$ ).

\section{Changes in psychological demand}

The majority of climbs in our research were top ropings to control the level of emotional arousal. Top roping is an ascent style in which the climber does not need to attend to the safety rope; thus, the participants could concentrate on the physical moves in a secure setting. However, in the second part of phase III, our climbers led repetition of the difficult route. This change in the safety rope protocol was used to increase the stress and helped determine the effect of psychological demand (III-ER $\mathrm{TR}_{\mathrm{TR}} / \mathrm{III}-\mathrm{DR}_{\mathrm{L}}$ ).

\section{Statistical analysis}

The obtained results are expressed as mean \pm standard deviation $(S D)$. Comparisons of 2 variables (testretest, easy and difficult route, 1 climb and 3 repetitions, top rope climb and lead climb) were carried out with the Wilcoxon matched pairs test. The Spearman's rank correlation was used for ordinal scale variables. The level of statistical significance was set at $p<0.05$. A reliability analysis was performed to determine the reliability of the $\mathrm{NASA}_{\text {climbing }}$ inventory, and Cronbach's alpha reliability coefficient was calculated. The data analysis tool was Statistica 12.0 (StatSoft Poland, 2015).

\section{Ethical approval}

The research related to human use has been complied with all the relevant national regulations and institutional policies, has followed the tenets of the Declaration of Helsinki, and has been approved by the authors' institutional review board or an equivalent committee.

\section{Informed consent}

Informed consent has been obtained from all individuals included in this study.

\section{Results}

Table 1 presents the details of HR and HRV measurements while Table 2 shows the results of the psychological inventories used in the study.

\section{Change in physical workload}

Route difficulty (physical workload) increased by decreasing the hold size ( 3 vs. 1 phalanx and 4 vs. 2 fingers). The increase in route difficulty was reflected in HR (CL-HR peak $_{1} 136$ for II-ER $\mathrm{TR}_{\mathrm{TR}}$ vs. 142 for II-DR $\mathrm{TR}_{\mathrm{TR}}$ ) (Figure 2). Parasympathetic activation (P-lnHF) was

Table 1. Absolute values of HR and HRV on 2 separate days (phases II and III) measured before and during the easy and difficult routes (physical workload increase), with long and short recovery (effect of fatigue), in top rope and lead climbs (change in psychological demand)

\begin{tabular}{lcccccc} 
& II-ER $_{\mathrm{TR}}$ & II-DR $_{\mathrm{TR}}$ & II-DR $_{\mathrm{TR} 3}$ & III-ER $_{\mathrm{TR}}$ & III-DR $_{\mathrm{TR}}$ & III-DR $_{\mathrm{L}}$ \\
\hline CL-HR $_{\text {peak }}(\mathrm{b} / \mathrm{min})$ & $136 \pm 15$ & $142 \pm 13^{\mathrm{b}}$ & $148 \pm 14^{\mathrm{c}}$ & $133 \pm 9$ & $140 \pm 11$ & $148 \pm 12^{\mathrm{d}}$ \\
CL-HR $_{\text {ave }}(\mathrm{b} / \mathrm{min})$ & $126 \pm 14$ & $129 \pm 13$ & $133 \pm 12$ & $122 \pm 8^{\mathrm{a}}$ & $129 \pm 11$ & $136 \pm 11^{\mathrm{d}}$ \\
CL-HR $_{\text {start }}(\mathrm{b} / \mathrm{min})$ & $104 \pm 16$ & $97 \pm 17^{\mathrm{b}}$ & $92 \pm 14$ & $91 \pm 5^{\mathrm{a}}$ & $97 \pm 12$ & $99 \pm 16$ \\
P-HR $_{\text {ave }}(\mathrm{b} / \mathrm{min})$ & $86 \pm 12$ & $78 \pm 9^{\mathrm{b}}$ & $76 \pm 9$ & $83 \pm 11$ & $80 \pm 11$ & $80 \pm 10$ \\
P-lnLF $\left(\mathrm{ms}^{2}\right)$ & $6.66 \pm 0.65$ & $6.83 \pm 0.53$ & $6.88 \pm 0.74$ & $6.73 \pm 0.70$ & $6.82 \pm 0.92$ & $7.31 \pm 0.61^{\mathrm{d}}$ \\
P-lnHF $\left(\mathrm{ms}^{2}\right)$ & $5.42 \pm 1.00$ & $5.78 \pm 0.70^{\mathrm{b}}$ & $6.11 \pm 0.73^{\mathrm{c}}$ & $5.68 \pm 1.00$ & $5.81 \pm 0.92$ & $5.94 \pm 0.88$ \\
P-lnTP $\left(\mathrm{ms}^{2}\right)$ & $7.07 \pm 0.68$ & $7.23 \pm 0.57$ & $7.42 \pm 0.68^{\mathrm{c}}$ & $7.16 \pm 0.71$ & $7.28 \pm 0.82$ & $7.67 \pm 0.65^{\mathrm{d}}$ \\
P-LF/HF & $4.30 \pm 2.14$ & $3.06 \pm 0.84^{\mathrm{b}}$ & $3.01 \pm 2.21$ & $3.60 \pm 2.24$ & $3.37 \pm 2.06$ & $4.71 \pm 2.39^{\mathrm{d}}$ \\
\hline
\end{tabular}

$\mathrm{HR}$ - heart rate, HRV - heart rate variability, II, III - phases of study, $\mathrm{ER}_{\mathrm{TR}}$ - easy route, top rope climb, $\mathrm{DR}_{\mathrm{TR}}-\mathrm{difficult}_{\mathrm{foute}}$, top rope climb, $\mathrm{DR}_{\mathrm{TR} 3}$ - difficult route, top rope climb, 3 repetitions, $\mathrm{DR}_{\mathrm{L}}$ - difficult route, lead climb,

CL - climb, P - pre-climb, LF - low frequency,

$\mathrm{HF}$ - high frequency, TP - total power

${ }^{a} p<0.05$, test-retest (II-ER $\mathrm{TR}_{\mathrm{TR}}$ vs. III-ER $\mathrm{TR}_{\mathrm{TR}}$, II-DR $\mathrm{DR}_{\mathrm{TR}}$ vs. III-DR $\mathrm{DR}_{\mathrm{TR}}$ )

${ }^{\mathrm{b}} p<0.05$, increase in route difficulty (II-ER $\mathrm{ER}_{\mathrm{TR}} \mathrm{vs}$. II-DR $\left.\mathrm{DR}_{\mathrm{TR}}\right)$

${ }^{\mathrm{c}} p<0.05$, effect of 5 -minute recovery after 3 repetitions (II-DR $\mathrm{TR}_{\mathrm{TR}} \mathrm{Vs}$. II-DR $\mathrm{TR}_{\mathrm{TR} 3}$ )

${ }^{\mathrm{d}} p<0.05$, top rope $\rightarrow$ lead climb (III-DR $\mathrm{TR}_{\mathrm{TR}}$ vs. III-DR $\mathrm{L}$ ) 
A. Magiera, I. Łukasik, R. Roczniok, O. Placek, Psychophysiological responses in climbers

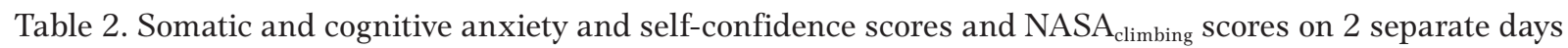
(phases II and III) measured before and after the easy and difficult routes (change in physical workload), with long and short recovery (effect of fatigue), in top rope and lead climbs (change in psychological demand)

\begin{tabular}{lcccccc} 
& \multicolumn{1}{c}{$\mathrm{II}^{-\mathrm{ER}_{\mathrm{TR}}}$} & \multicolumn{1}{c}{$\mathrm{II}-\mathrm{DR}_{\mathrm{TR}}$} & $\mathrm{II}-\mathrm{DR}_{\mathrm{TR} 3}$ & III-ER $_{\mathrm{TR}}$ & III-DR $_{\mathrm{TR}}$ & III-DR $_{\mathrm{L}}$ \\
\hline Asom & $12.50 \pm 3.26$ & $12.20 \pm 2.79$ & $12.26 \pm 3.13$ & $11.79 \pm 2.21$ & $12.14 \pm 2.95$ & $12.74 \pm 3.63$ \\
Acog & $15.00 \pm 4.05$ & $15.17 \pm 4.78$ & $15.50 \pm 4.98$ & $13.67 \pm 3.80$ & $14.33 \pm 4.74$ & $17.00 \pm 5.15^{\mathrm{d}}$ \\
Scon & $29.67 \pm 4.42$ & $30.00 \pm 4.51$ & $29.00 \pm 4.63$ & $29.83 \pm 5.15$ & $28.33 \pm 4.96$ & $26.83 \pm 4.78$ \\
NASA $_{\text {climbing }}$ & $31.03 \pm 11.95$ & $36.89 \pm 18.56^{\mathrm{b}}$ & $39.86 \pm 16.07$ & $27.61 \pm 11.89$ & $34.36 \pm 15.44$ & $43.53 \pm 18.13^{\mathrm{d}}$ \\
Mental & $5.17 \pm 3.88$ & $6.25 \pm 4.75$ & $5.50 \pm 3.66$ & $4.42 \pm 3.45$ & $5.08 \pm 4.10$ & $7.25 \pm 3.70^{\mathrm{d}}$ \\
Strength & $4.00 \pm 2.30$ & $6.50 \pm 3.53^{\mathrm{b}}$ & $8.17 \pm 3.93^{\mathrm{c}}$ & $3.67 \pm 1.72$ & $6.83 \pm 3.43$ & $7.00 \pm 3.74$ \\
Temporal & $3.75 \pm 2.60$ & $3.83 \pm 2.33$ & $3.83 \pm 2.72$ & $3.25 \pm 1.54$ & $4.25 \pm 3.44$ & $9.42 \pm 4.80^{\mathrm{d}}$ \\
Satisfaction & $9.50 \pm 5.54$ & $9.67 \pm 5.94$ & $10.83 \pm 5.62$ & $7.50 \pm 5.20^{\mathrm{a}}$ & $7.75 \pm 5.50^{\mathrm{a}}$ & $10.33 \pm 5.73^{\mathrm{d}}$ \\
Endurance & $4.00 \pm 1.65$ & $6.50 \pm 3.90^{\mathrm{b}}$ & $8.00 \pm 3.25^{\mathrm{c}}$ & $4.67 \pm 2.06$ & $6.58 \pm 2.19$ & $6.92 \pm 2.75$ \\
Frustration & $2.50 \pm 1.83$ & $2.58 \pm 1.44$ & $3.00 \pm 1.48$ & $2.50 \pm 1.62$ & $2.25 \pm 1.06$ & $4.33 \pm 3.52$ \\
\hline
\end{tabular}

II, III - phases of study, $\mathrm{ER}_{\mathrm{TR}}$ - easy route, top rope climb, $\mathrm{DR}_{\mathrm{TR}}-$ difficult route, top rope climb, $\mathrm{DR}_{\mathrm{TR} 3}-$ difficult route, top rope climb, 3 repetitions, $\mathrm{DR}_{\mathrm{L}}$ - difficult route, lead climb, Asom - somatic anxiety, Acog - cognitive anxiety,

Scon - self-confidence

${ }^{\mathrm{a}} p<0.05$, test-retest (II-ER $\mathrm{TR}_{\mathrm{TR}}$ vs. III-ER $\mathrm{ER}_{\mathrm{TR}}$, II-DR $\mathrm{DR}_{\mathrm{TR}}$ vs. III-DR $\left.\mathrm{DR}_{\mathrm{TR}}\right)$

${ }^{\mathrm{b}} p<0.05$, increase in route difficulty (II-ER $\mathrm{ER}_{\mathrm{TR}} \mathrm{vs}$. II-DR $\left.\mathrm{DR}_{\mathrm{TR}}\right)$

${ }^{\mathrm{c}} p<0.05$, effect of 5 -minute recovery after 3 repetitions (II-DR $\mathrm{TR}_{\mathrm{TR}} \mathrm{vs}$. II-DR $\left.\mathrm{TR}_{\mathrm{TR} 3}\right)$

${ }^{\mathrm{d}} p<0.05$, top rope $\rightarrow$ lead climb (III-DR $\mathrm{TR}_{\mathrm{T}}$ vs. III-DR ${ }_{\mathrm{L}}$ )

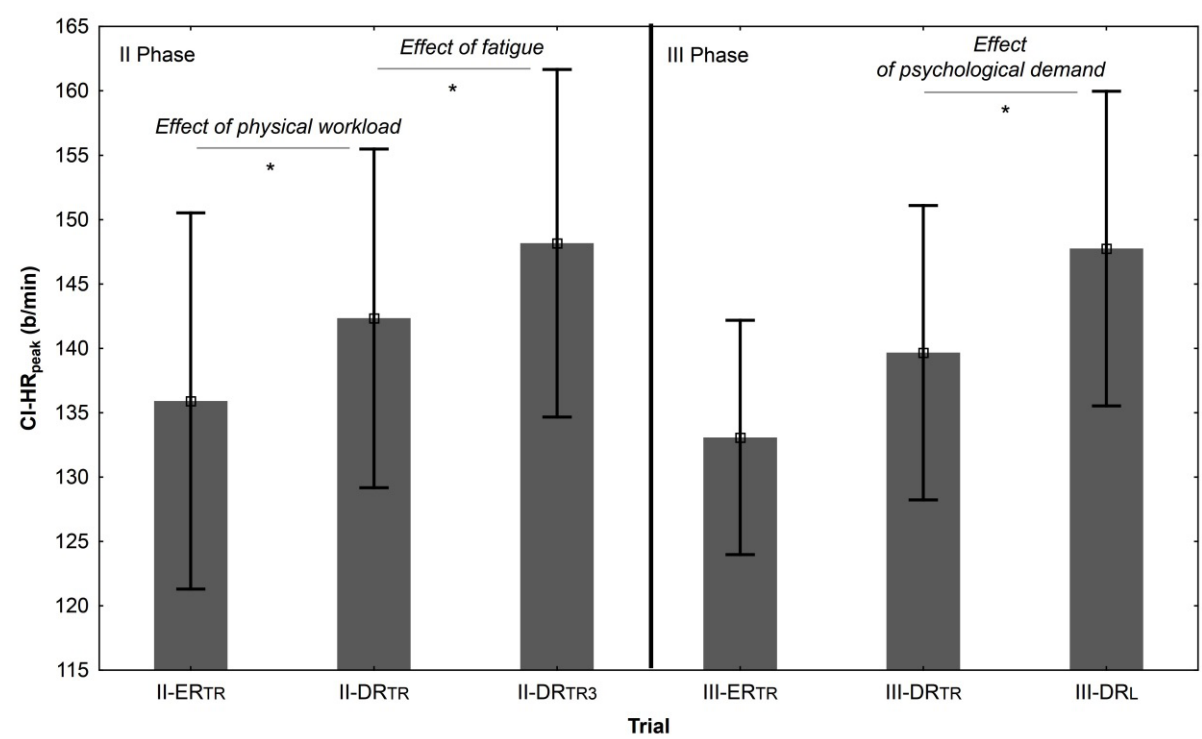

II, III - phases of study, $\mathrm{ER}_{\mathrm{TR}}$ - easy route, top rope climb, $\mathrm{DR}_{\mathrm{TR}}$ - difficult route, top rope climb, $\mathrm{DR}_{\mathrm{TR} 3}$ - difficult route, top rope climb, 3 repetitions,

$\mathrm{DR}_{\mathrm{L}}$ - difficult route, lead climb

${ }^{*} p<0.05$, significant difference compared with the preceding climbing task

Figure 2. Changes in heart rate peak $\left(\mathrm{Cl}-\mathrm{HR}_{\text {peak }}\right)$ measured during the easy and difficult routes (physical workload increase), with long and short recovery (effect of fatigue), in top rope and lead climbs (change in psychological demand)

less pronounced prior to the easy route. No similar response was noted with respect to $\mathrm{CL}-\mathrm{HR}_{\text {ave }}, \mathrm{P}-\mathrm{lnLF}$, or P-lnTP. The following variables decreased before the difficult route: CL-HR ${ }_{\text {start }}, \mathrm{P}_{-} \mathrm{HR}_{\text {ave }}$, and P-LF/HF. Prior to the climb, no differences were observed with respect to state anxiety or self-confidence. Higher Strength, Endurance, and total $\mathrm{NASA}_{\text {climbing }}$ scores were obtained after the completion of the difficult route (NASA $_{\text {climbing: }} 31$ for II-ER TR $_{\mathrm{TR}}$ vs. 37 for II-DR ${ }_{\mathrm{TR}}$ ).

\section{Effect of fatigue}

Shortening of the recovery period from approximately 30 to 5 minutes caused a difference in fatigue. Fatigue accumulation after 3 repetitions of the difficult route had an effect on CL-HR peak $\left(142\right.$ for II-DR ${ }_{\mathrm{TR}}$ vs. 148 for II-DR ${ }_{\mathrm{TR} 3}$ ) (Figure 2). CL-HR elevation was caused by a gradual increase in $\mathrm{P}-\mathrm{HR}_{\text {ave }}$ and CL-HR $\mathrm{H}_{\text {start }}$ as measured during each recovery in a 3-repetition 

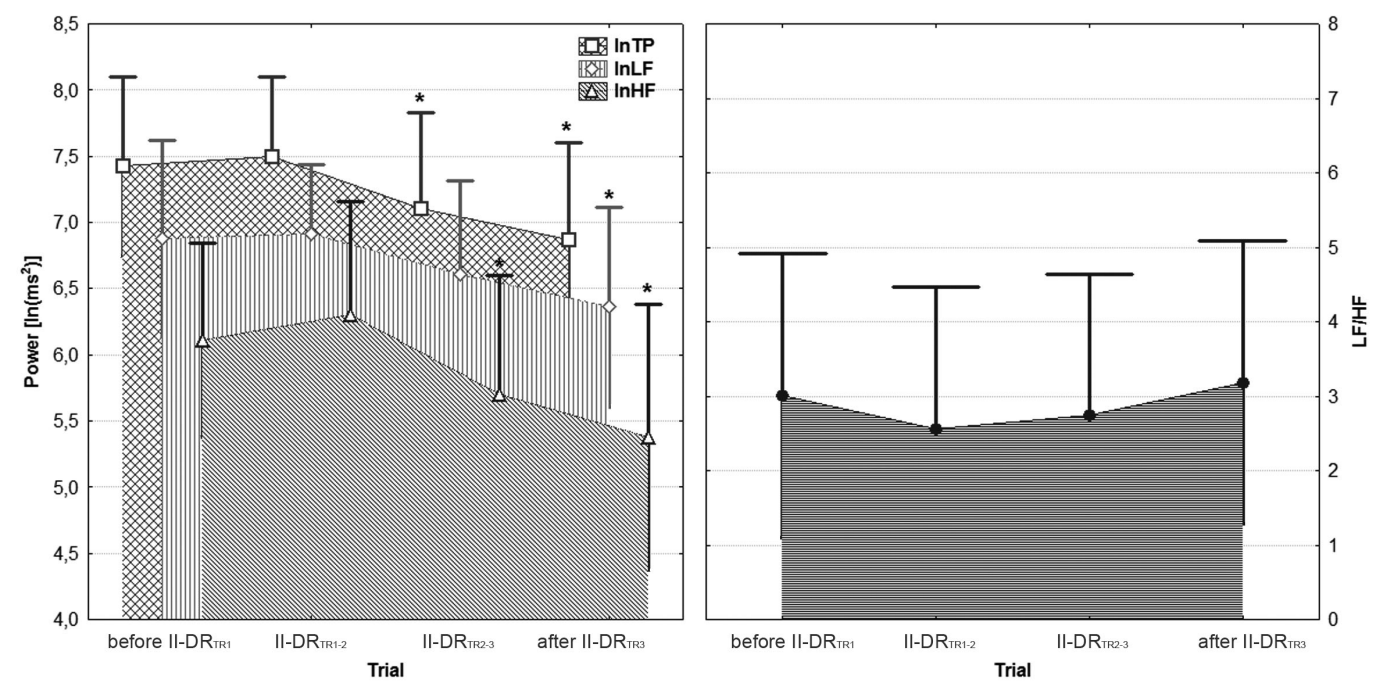

TP - total power, LF - low frequency, HF - high frequency, II - phase II of study, $\mathrm{DR}_{\mathrm{TR} 1}-\mathrm{DR}_{\mathrm{TR} 3}-$ difficult route, top rope climb, 3 repetitions ${ }^{*} p<0.05$, significant difference compared with the preceding climbing task

Figure 3. Changes in lnTP, lnLF, lnHF powers (left graph) and LF/HF (right graph) in the series of 3 repetitions of II-DR with 5-minute recovery periods

series. The lack of differences in resting HR (CL-HR start $_{\text {, }}$ P-HR ${ }_{\text {ave }}$ ) and several HRV variables (P-lnLF, P-LF/HF) indicates that the conditions of each trial were comparable (however, differences were noted regarding P-lnHF, P-lnTP). During the recovery periods between climbing repetitions, the effect of the pre-existing workload was evidenced by a gradual decrease in HRV (TP, LF, HF) with no marked changes in sympathetic influences (LF/HF) (Figure 3).

Higher Strength and Endurance scores indicate higher fatigue after 3 repetitions. The overall perceived workload score was also higher $\left(\mathrm{NASA}_{\text {climbing }}: 37\right.$ for II-DR ${ }_{\mathrm{TR}}$ vs. 40 for II-DR $\mathrm{TR}_{\mathrm{T} 3}$ ) but did not reach the level of statistical significance. The psychological load did not differ between the single and serial climbing task (Table 2; II-DR $\mathrm{TR}_{\mathrm{TR}}$ vs. II-DR $\mathrm{TR}_{\mathrm{T} 3}$ ).

\section{Change in psychological demand}

Lead climb was associated with higher HR values (CL-HR ${ }_{\text {ave }}: 129$ for III-DR $\mathrm{TR}_{\mathrm{TR}}$ vs. 136 for III-DR $\mathrm{L}_{\mathrm{L}}$ CL-HR $\mathrm{Ceak}_{\text {pea }}$ : 140 for III-DR $\mathrm{TR}_{\mathrm{TR}}$ vs. 148 for III-DR $\mathrm{L}_{\mathrm{L}}$ ) (Figure 2). No differences related to change in safety rope protocol were noted in resting $\mathrm{HR}$ values $\left(\mathrm{P}-\mathrm{HR}_{\text {ave }}, \mathrm{CL}-\mathrm{HR}_{\text {start }}\right)$. The high variability of individual differences in CL-HR $\mathrm{H}_{\text {start }}$ might have been associated with climbing experience as these 2 variables were strongly negatively correlated $(n=12, R=-0.77, p<0.01)$. Significant pre-lead climb differences in P-lnLF, P-lnTP, P-LF/HF might indicate the predominance of sympathetic activation. Experience and P-LF/HF were also correlated $(n=12$, $R=-0.58, p<0.05$ ).
Cognitive anxiety was higher prior to lead climb (Acog: 14.33 for III-DR $\mathrm{TR}_{\mathrm{TR}}$ vs. 17.00 for III-DR $\mathrm{L}_{\mathrm{L}}$ ). State anxiety (CSAI-2R) was the most highly correlated with trait anxiety (the correlations of SCAT with Acog, Asom, and Scon: $R=0.63, p<0.05 ; R=0.68, p<0.05$; and $R=-0.7, p<0.05$, respectively; $n=12$ ). CSAI- $2 \mathrm{R}$ parameters were also correlated with satisfaction level (Satisfaction: $R=-0.3, p<0.05$ for Acog; $R=-0.51$, $p<0.001$ for Asom; $R=0.41, p<0.001$ for Scon) and frustration level (Frustration: $R=0.31, p<0.01$ for Acog; $R=0.33, p<0.01$ for Asom; $R=-0.31, p<0.01$ for Scon). The scores on mental demand (5.08 for III$\mathrm{DR}_{\mathrm{TR}}$ vs. 7.25 for III-DR $\mathrm{L}_{\mathrm{L}}$ ), temporal demand (4.25 for III-DR ${ }_{T R}$ vs. 9.42 for III-DR ${ }_{\mathrm{L}}$ ), and satisfaction level (7.75 for III-DR ${ }_{\mathrm{TR}}$ vs. 10.33 for III-DR ${ }_{\mathrm{L}}$ ) were all higher after lead climb. Consequently, the total workload score was also higher $\left(\mathrm{NASA}_{\text {climbing: }} 34\right.$ for III-DR $\mathrm{DR}_{\mathrm{TR}}$ vs. 44 for III-DR $\mathrm{L}_{\mathrm{L}}$.

\section{Climbing test-retest}

In order to check the reliability of the research tools, we compared HR and HRV measurements on the easy and difficult top rope routes during phases II and III (Table 1; test-retest). There were no statistically significant differences between phase II and phase III results on the difficult route. HR and HRV variables of phase II and III easy route were also similar, except for CL-HR start (104 vs. 91 beats/min) and CL-HR (126 vs. 122 beats/min). Among the subjective variables, only the level of satisfaction with completion of both easy and difficult routes was lower in phase III. 
A. Magiera, I. Łukasik, R. Roczniok, O. Placek, Psychophysiological responses in climbers

The environmental conditions were similar in phases II and III. Measurements started around 08:45 hours $( \pm 45 \mathrm{~min})$, at the temperature of around $19^{\circ} \mathrm{C}$ (18.9-19.7), 49\% humidity (44-52), and atmospheric pressure of around $977 \mathrm{hPa}$ (966-985).

\section{Verification of $\mathrm{NASA}_{\text {climbing }}$}

The reliability of NASA $\mathrm{N}_{\text {climbing }}$ was first checked on the basis of Cronbach's alpha coefficient. The Polish version of NASA-TLX had the Cronbach's alpha of 0.760.77 [15]. In our study, the 6 subscales of NASA climbing $_{\text {- }}$ had Cronbach's alpha of 0.76 ; therefore, they could be used as acceptable measures of the studied construct.

\section{Discussion}

In our research, changes in the physical workload, short-term fatigue, and psychological demand of climbing influenced HR and HRV, anxiety (CSAI-2R), and perceived climbing load (NASA climbing $_{\text {) }}$ in different ways. The impact was observed in the pre-exercise phase and during climbing.

\section{Heart rate}

Highly dynamic variations of HR were observed during the experiment. The HR and HRV measurement was accurate and reliable, which was confirmed during the retest day. CL-HR peak was used in further workload analyses since the short duration of the climb and slight latency of HR response to an increase in muscle work had an effect on CL-HR ave $_{\text {. }}$

We measured the levels of HR and HRV immediately before the climb during several climbing tasks. Unexpectedly, the pre-climb arousal was higher before the easy compared with the difficult route (higher CL-HR $\mathrm{H}_{\text {start }}$, lower parasympathetic and higher sympathetic activation). The uncertainty before the first climb of phase II could have been the cause although the climbers did get acquainted with the climbing route and study procedure in phase I. This hypothesis seems to be confirmed by the absence of similar differences in phase III, as well as lack of differences in pre-climb anxiety and self-confidence levels.

Compared with the pre-climb status, the most noticeable differences in HR and HRV were observed following the change in the safety rope protocol. The pre-climb arousal was higher before lead climb compared with top roping, but it depended on climbing experience. Sympathetic activation was increased (LF/HF, lnLF), resulting in an increase of overall au- tonomic activity (lnTP). However, more experienced climbers exhibited lower sympathetic activation and HR compared with those with less experience. Our results seem to confirm the conclusion by Fryer et al. [10], who emphasized the complexity of factors that affect the pre-climb status, including climbing experience, style (e.g. OS, RP), and grade of a climbing route. For example, advanced performers did not find lead climbing more stressful than top rope climbing during the OS ascent.

A change in safety rope protocol may also affect HR during climbing [8-10]. However, such change typically resulted in an increase in tope roping velocity, which itself could have elicited higher HR values [16]. The only variable in our study was the change in safety rope protocol; the other experimental conditions remained the same. In a study by Draper et al. [8], the difference in lead climb and top roping HR values was 10 beats/min ( 175 and 165 beats/min, respectively), but did not reach the level of statistical significance. In our study, the difference in lead and top rope HR values was comparable to that observed in the above mentioned study and amounted to 8 beats/min; however, it was statistically significant. We suggest that the excessive HR increase observed in our intermediate climbers during lead climbing was mainly associated with anxiety and increase in sympathetic activation prior to climbing.

The lifting force on shallow holds becomes reduced while the difficulty of climbing moves increases. The same happens when fewer fingers are placed into a pocket. In our study, the increase in route difficulty

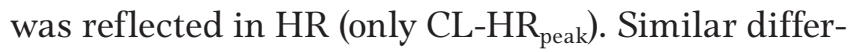
ences in heart work were observed in other studies on HR during climbing routes of varying difficulty [17-21]. Burnik and Jereb [21] compared 15-second HR peaks during OS climbing on $4 \mathrm{c}, 5 \mathrm{c}$, and $6 \mathrm{~b}$ routes; the respective HR values were $142 \pm 22,156 \pm 19$, and $163 \pm 21$ beats/min; the differences were statistically significant. Our results also revealed inter-route

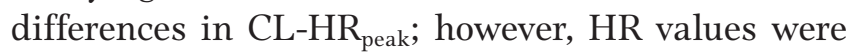
lower $\left(136 \pm 15\right.$ for II-ER ${ }_{\mathrm{TR}}$ vs. $142 \pm 13$ for II-DR $\left.{ }_{\mathrm{TR}}\right)$ owing to lower technical difficulty (a single move type) and lower mental demand (familiarity with the climbing route).

Other factors affecting the effort associated with route completion were also analysed, including climbing velocity [16], overhangs [22], route characteristics [23, 24], climbing style [4], and climbing level [10, 25]. For example, it was found that HR increased linearly with route difficulty but the increase was nonproportional to $\mathrm{VO}_{2}$ increases (this was accounted for 
by anaerobic-aerobic metabolism and mental arousal). Except for physical workload and psychological demand, we also analysed a third factor, i.e., fatigue, which was

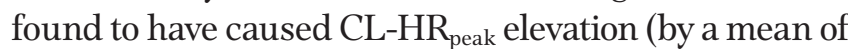
6 beats/min) and a decrease in HRV, with no marked changes in sympathetic influences. This lower activation of the parasympathetic nervous system during stable conditions (rest period) might indicate fatigue while sympathetic increase might point at psychological arousal and anxiety.

\section{Pre-climb anxiety}

There are several scientific theories on the effect of pre-exercise arousal on physiological responses and the efficiency of subsequent exercise. Research into sport rock climbing is mostly based on the multidimensional anxiety theory of Martens [8-10, 26], who introduced the notion of competitive anxiety, a specific form of anxiety associated with sport performance. Martens et al. [27] distinguished between competitive trait anxiety (a relatively inherent tendency to perceive competitive situations as threatening) and competitive state anxiety (arousal and tension in response to a particular stimulus, comprised of a cognitive component and a somatic component).

We measured the levels of cognitive and somatic anxiety and self-confidence immediately before the climb during several climbing tasks. The most noticeable differences were observed with respect to the preclimb status following the change in the safety rope protocol. Cognitive anxiety was higher before lead climb compared with top roping. This confirmed that the change in safety rope protocol (top rope $\rightarrow$ lead climb) caused mental stress in intermediate climbers.

Previous research among intermediate-level climbers revealed differences in CSAI-2R scores. In a study by Hodgson et al. [9], climbers completed lead, top-rope, and lead and top-rope climbs. Repeated measures ANOVA showed significant differences for somatic anxiety and self-confidence scores but not for cognitive anxiety. Draper et al. [8] and Fryer et al. [10] did not find significant differences in CSAI-2R scores obtained for top rope and lead climbs. We had contrary results; cognitive anxiety levels were different for the above mentioned safety rope protocols. Also, mean scores in somatic anxiety, cognitive anxiety, and selfconfidence were lower compared with those obtained in the above mentioned studies (Table 2), which might indicate that several repetitions of a route and a single pre-set move were a less difficult challenge for the climbers. Additionally, pre-climb state anxiety and self- confidence were affected by trait anxiety (SCAT), which is consistent with the theory of Martens.

\section{Post-climb perceived workload}

At present, route difficulty (RSD) is subjectively determined by the first ascensionist [3]. We aimed to make this determination more objective and based on physiological response to physical effort (HR) and psychological measurements ( $\mathrm{NASA}_{\text {climbing }}$ ).

A decrease in hold size is a method of raising route difficulty (RSD). The increase in route difficulty was reflected in perceived climbing workload. The modified NASA-TLX test for climbing $\left(\mathrm{NASA}_{\text {climbing }}\right)$ effectively showed differences in physical load (higher Strength, Endurance, and total NASA $\mathrm{Alimbing}_{\text {scores). }}$ Increased fatigue during exercise and insufficient rest produce a perception of higher load. NASA climbing $_{\text {proved }}$ a good tool to measure the effects of fatigue. Higher Strength and Endurance scores indicated greater physical workload after 3 repetitions of the climbing task.

A change in safety rope protocol also affected perceived climbing load. Draper et al. [8] concluded that lead climb was mentally more demanding than top rope climb. The NASA-TLX scores indicated significant differences in mental demand $(11 \pm 4$ for lead climb vs. $9 \pm 4$ for top rope climb), physical demand (13 \pm 3 vs. $8 \pm 4$ ), effort (13 \pm 4 vs. $9 \pm 5$ ), and frustration (10 \pm 5 vs. $5 \pm 3$ ). In our study, post-lead NASA climbing scores were lower. Pre-set climbing velocity resulted in an increase in psychological load only, i.e., mental demand (consistent with the above mentioned study), temporal demand, and satisfaction level. These results confirm that the NASA $\mathrm{Alimbing}_{\text {test }}$ effectively differentiates between physical and psychological demands of a climbing task.

Since our study only examined some of the multiple aspects of climbing performance, it has several limitations that warrant discussion. The main limitation is that all subjects were intermediate-level climbers and it is not possible to generalize the study results to advanced, more experienced climbers. Next, we attempted to control for other variables; hence, the workload model was quite simple (the use of technical and tactical skills was limited to one climbing move, constant climbing velocity, etc.) and remained in contrast to the diversity of climbing.

\section{Conclusions}

Physiological response (HR and HRV), perceived anxiety, and workload differed depending on the type 
A. Magiera, I. Łukasik, R. Roczniok, O. Placek, Psychophysiological responses in climbers

of the climbing task. Pre-climb status was mainly affected by the change in the safety rope protocol (top rope climb $\rightarrow$ lead climb), with increase in cognitive anxiety and sympathetic activation depending on climbing experience. This finding shows that lead climbing can be a stressful situation for intermediate climbers. HRV parameters can also indicate an increase in fatigue through a decrease in parasympathetic activation during the rest period. $\mathrm{NASA}_{\text {climbing }}$ scores effectively reflected differences in physical load (physical demand - strength and endurance) and psychological load (higher mental demand, temporal demand, and satisfaction level). However, the best parameter to evaluate climbing workload and cumulative psychophysiological climbing stress was CL-HR peak, which was correlated with physical workload, psychological demand, and increasing fatigue. It can thus be used to monitor climbing stress in a more objective manner. Additionally, we verified the reliability and accuracy of the NASA-TLX modification (NASA climbing $_{\text {) }}$ and the usefulness of HR and HRV measurements in climbing.

\section{Disclosure statement}

No author has any financial interest or received any financial benefit from this research.

\section{Conflict of interest}

The authors state no conflict of interest.

\section{References}

1. Doran DA, Moncrieff J. Energy expenditure and metabolic cost during technically similar but physically different rock climbing task. J Sports Sci. 1999;17(7):571609.

2. Watts PB. Physiology of difficult rock climbing. Eur J Appl Physiol. 2004;91(4):361-372; doi: 10.1007/s00421003-1036-7.

3. Delignières D, Famose J-P, Thépaut-Mathieu C, Fleurance $\mathrm{P}$. A psychophysical study of difficulty rating in rock climbing. Int J Sport Psychol. 1993;24:404-416.

4. Draper N, Jones GA, Fryer S, Hodgson C, Blackwell G. Effect of an on-sight lead on the physiological and psychological responses to rock climbing. J Sports Sci Med. 2008;7(4):492-498.

5. Michailov ML. Workload characteristic, performance limiting factors and methods for strength and endurance training in rock climbing. Med Sport. 2014;18(3):97106; doi: 10.5604/17342260.1120661.

6. Gallotta MC, Emerenziani GP, Monteiro MD, Iasevoli L, Iazzoni S, Baldari C, et al. Psychophysical benefits of rock-climbing activity. Percept Mot Skills. 2015;121(3): 675-689; doi: 10.2466/30.PMS.121c26x9.

7. Williams ES, Taggart P, Carruthers M. Rock climbing: observations on heart rate and plasma catecholamine concentrations and the influence of oxprenolol. Br J Sports Med. 1978;12(3):125-128; doi: 10.1136/bjsm. 12.3.125.

8. Draper N, Jones GA, Fryer S, Hodgson CI, Blackwell G. Physiological and psychological responses to lead and top rope climbing for intermediate rock climbers. Eur J Sport Sci. 2010;10(1):13-20; doi: 10.1080/17461390 903108125.

9. Hodgson CI, Draper N, McMorris T, Jones G, Fryer S, Coleman I. Perceived anxiety and plasma cortisol concentrations following rock climbing with differing safety rope protocols. Br J Sports Med. 2009;43(7):531535; doi: 10.1136/bjsm.2007.046011.

10. Fryer S, Dickson T, Draper N, Blackwell G, Hillier S. A psychophysiological comparison of on-sight lead and top rope ascents in advanced rock climbers. Scand J Med Sci Sports. 2013;23(5):645-650; doi: 10.1111/ j.1600-0838.2011.01432.x.

11. Achten J, Jeukendrup AE. Heart rate monitoring: applications and limitations. Sports Med. 2003;33(7): 517-538; doi: 10.2165/00007256-200333070-00004.

12. Weippert M, Kumar M, Kreuzfeld S, Arndt D, Rieger A, Stoll R. Comparison of three mobile devices for measuring R-R intervals and heart rate variability: Polar S810i, Suunto t6 and an ambulatory ECG system. Eur J Appl Physiol. 2010;109(4):779-786; doi: 10.1007/ s00421-010-1415-9.

13. Task Force of the European Society of Cardiology and the North American Society of Pacing and Electrophysiology. Heart rate variability: standards of measurement, physiological interpretation, and clinical use. Circulation. 1996;93(5):1043-1065; 10.1161/01.CIR.93.5.1043.

14. Borek-Chudek D. Anxiety levels and achievements of badminton players in light of martens multidimentional anxiety theory [in Polish]. Polish J Sport Med. 2007;3(6):140-148.

15. Biernacki M, Bicka-Capała M, Tarnowski A. Theoretical and methodological problems of workload testing on the example of the subjective workload assessment technique [in Polish]. Pol Przegl Med Lotn. 2007;13(4): 465-479.

16. Booth J, Marino F, Hill C, Gwinn T. Energy cost of sport rock climbing in elite performers. Br J Sports Med. 1999;33(1):14-18; doi: 10.1136/bjsm.33.1.14.

17. Sheel AW, Seddon N, Knight A, McKenzie DC, Warburton DE. Physiological responses to indoor rockclimbing and their relationship to maximal cycle ergometry.Med SciSportsExerc. 2003;35(7):1225-1231; doi: 10.1249/01.MSS.0000074443.17247.05.

18. Bertuzzi RC, Franchini E, Kiss MA. Heart rate and handgrip acute adjustments during the practice of the indoor rock climbing [in Portuguese]. Rev Mackenzie Educ Fis Esporte. 2004;3(3):99-106.

19. Bertuzzi RC, Franchini E, Kokubun E, Kiss MA. Energy system contributions in indoor rock climbing. Eur J Appl Physiol. 2007;101(3):293-300; doi: 10.1007/s00421007-0501-0. 
20. Hardy L, Hutchinson A. Effects of performance anxiety on effort and performance in rock climbing: a test of processing efficiency theory. Anxiety Stress Coping. 2007;20(2):147-161; doi: 10.1080/10615800701217035.

21. Burnik S, Jereb B. Heart rate as an indicator of sport climbing intensity. Acta Univ Palacki Olomuc Gymn. 2007;37(1):63-66.

22. Köstermeyer G. Determination, significance and training of the local strength endurance of finger flexors in sport climbing [in German]. Neuried: Ars Una; 2000.

23. Billat V, Palleja P, Charlaix T, Rizzardo P, Janel N. Energy specificity of rock climbing and aerobic capacity in competitive sport rock climbers. J Sports Med Phys Fitness. 1995;35(1):20-24.

24. De Geus B, O’Driscoll SV, Meeusen R. Influence of climbing style on physiological responses during indoor rock climbing on routes with the same difficulty. Eur J Appl Physiol. 2006;98(5):489-496; doi: 10.1007/ s00421-006-0287-5.

25. Janot JM, Steffen JP, Porcari JP, Maher MA. Heart rate responses and perceived exertion for beginner and recreational sport climbers during indoor climbing. J Exerc Physiol Online. 2000;3(1):1-7.

26. Sanchez X, Boschker MS, Llewellyn DJ. Pre-performance psychological states and performance in an elite climbing competition. Scand J Med Sci Sports. 2010; 20(2):356-363;doi:10.1111/j.1600-0838.2009.00904.x.

27. Martens R, Vealey RS, Burton D. Competitive anxiety in sport. Champaign: Human Kinetics; 1990. 